\title{
Expansion of Hull Method to 3-Dimensional Crystal Anisotropic Etching Simulation
}

\author{
Meng Zhao, ${ }^{1 *}$ Jing $\mathrm{Ji}^{1}{ }^{1}$ and Toshitsugu $\mathrm{Ueda}^{2}$ \\ ${ }^{1}$ School of Electro-Mechanical Engineering, Xidian University, No. 2 South Taibai Road, Xi'an 710071, P.R. China \\ ${ }^{2}$ IPS Research Center, Waseda University, 2-2 Hibikino, Wakamatsu-ku, Kitakyushu, Fukuoka 808-0135, Japan
}

(Received December 1 2017; accepted May 8, 2018)

Keywords: crystal, etching, simulation, Hull method, 3-dimensional

We present here a new approach to simulating three-dimensional (3D) vertex etching, which is applicable to quartz crystals and other crystals. In this paper, we describe the theoretical method behind this approach and give details regarding processing the vertex, processing the edges, distinguishing four types of vertexes, and determining the etching depth of each vertex point.

\section{Introduction}

Quartz has been widely used as a material in micro-electromechanical systems (MEMS) because of its piezoelectric behavior, good frequency stability with temperature, and low power consumption. The development of a quartz anisotropic wet etching simulator is very important because it can guide the etching and greatly reduce the expense, thereby guiding the design of quartz MEMS devices. In the past thirty years, the geometric rules of the crystal etching process have been studied from different perspectives. ${ }^{(1-3)}$ Since then, many researchers have focused on developing simulators that can simulate crystal etching processes. Tellier et al.$^{(4,5)}$ developed a quartz three-dimensional (3D) simulator using a vector analysis method in 1986 and proposed the slow rate method for the first time. Since 1991, Sato et al. ${ }^{(6,7)}$ have made significant progress in the $3 \mathrm{D}$ anisotropic wet etching simulator. Their simulators (MICROCAD and FabMeister-ES) are suitable for silicon and quartz. While some other simulators only apply to silicon, ${ }^{(8-11)}$ almost all of these simulators either lack a high-accuracy etch rate database for quartz, or the results of quartz simulation are not good enough because of the nature of the mathematical tool used or other reasons. It can be said that the development of anisotropic wet etching for the study of quartz has been slow, supporting software is limited, the price is high, and the application range is limited. In order to provide a low-cost, high-performance quartz anisotropic wet etching simulator, we conducted research on quartz wet etching simulation. However, research on 3D simulators has been slow. Therefore, we have developed a highperformance, noncharged quartz 3D anisotropic etching simulator.

*Corresponding author: e-mail: mzhao@xidian.edu.cn http://dx.doi.org/10.18494/SAM.2018.1833 
The etching rate database of the simulator is novel and highly accurate. The Hull method used in this simulation is based on Sequin's work ${ }^{(12)}$ but improves upon it. This work itself, on the 3D anisotropic etching simulation of a vertex, is original. The level set method was another effective method used in shape evolution simulation, which was first proposed by Osher and Sethian ${ }^{(1)}$ in 1988. However, the authors think that for the special case of quartz wet etching, first of all, the original quartz configuration used for the fabrication of devices is mostly simple and has less curved surfaces. Secondly, the work of quartz omnidirectional etching speed determination started late. The accuracy of the etching rate database may not guarantee the accumulation of errors in the solution evolution in the level set method. From these two perspectives, we consider the Hull method to be more appropriate and sufficient for our research aim. The Hull method essentially describes the selection process of the dominant surface in the etching process using a geometric method. The Hull method requires less computing resources than the level set method, especially when used for a simple initial configuration and linear or planar evolution, which is most common in the wet etching of crystals.

This paper is part of a series in which we will report all of our progress on the $3 \mathrm{D}$ anisotropic etching simulator. In this paper, we focus on describing the efforts that we have made in five parts as shown in Sect. 2: how to decompose an object into units that can be processed, how to distinguish 3D corner types, how a corner vertex changes its shape during etching, how an edge changes its shape during etching, and finally, how to predict the etching direction and distance of a single vertex. In Sect. 3, we give a 3D simulation result compared with the experimental result as an example.

\section{Methods}

The database that we used in this work is the same as the database used in the twodimensional (2D) anisotropic etching simulator, ${ }^{(13)}$ which has been completed. The etching rates of quartz wafers with 32 different angles of cut were measured when etching with ammonium bifluoride $\left(\mathrm{NH}_{4} \mathrm{HF}_{2}\right)$ at $82{ }^{\circ} \mathrm{C}$. We considered the symmetry of the crystals and then used a 3D interpolation to form the slowness rate database.

The core of the geometric analysis method used in this simulator is Hull construction reported by Sequin in 1992. ${ }^{(3)}$ Hull construction is the most important step in anisotropic simulation-namely, the selection of a dominant etching direction. We extended this step into 3D.

The size and shape of the mask is an important factor in the etching, so we have to consider the application of different types of masks during the development of the simulator. In the 2D simulator, the mask is represented as a combination of $2 \mathrm{D}$ line segments. In the $3 \mathrm{D}$ case, our simulator is theoretically suitable for masks of arbitrary shape and size. However, for the sake of application convenience, the simulation interface for rectangular masks and circular masks will be given separately. In addition, there will be another simulation interface suitable for masks of arbitrary shapes and sizes. In the process of developing the simulation system, we encountered several theoretical difficulties, and their solutions are presented in the following. 


\subsection{Method of splitting 3D crystals into processable units}

The first problem that we encountered in the design of the entire quartz anisotropic etching simulation system is that we needed to divide the entire problem into a set of manageable small problems. For a variety of reasons, we chose the geometric simulation method. We first realized the inherent principle of crystal anisotropy, and then we mimicked the geometric process of crystal vanishing at various points, lines, and surfaces during the etching process. At this point, however, we still faced the choice of picking which point, line, or surfaces to use as our unit of decomposition. In the end, we chose a point, specifically the vertex point, as the basic unit, and a line, specifically the edge line, as an adjunct. The reason for these choices is based on the convenience of the mathematical description because, if the face is selected as the processing object after decomposition, although the evolution direction and distance of each face are very easy to determine, it is inevitable that one confronts the complicated problem of shape determination at a face-face interface.

Figure 1 shows an example of the image of the split process (of course, in the actual situation, we deal with the object that is not a simple cube). First, we select an object to be etched, namely, a cube. Then we conceptually split it into several pieces, that is, it is split into a number of pieces equal to the number of vertex points. In this example, we now have eight cells to process. Each vertex point is then evaluated for the direction of its evolution in subsequent steps. Its direction of evolution is generally in the opposite direction of the etchant, and it splits many times into multiple points, as shown. The points can be calculated. Finally, we combine all the evolving vertexes conceptually to get the shape of the cube after it has been etched. The calculation for each vertex point will be described later. This is just an example of a geometry whose vertexes are all convex vertexes, and in fact, we can handle a variety of vertexes, whether convex or concave in addition to all the other complex corners, as we will see later. These calculations are still just for one time step (the length of which can be set) in the entire simulation. What actually happens is that such steps need to be repeated until the desired duration or shape is reached.

\subsection{Method of distinguishing 3D corner types}

In this section, we discuss how to use mathematical language to describe and distinguish different types of vertex points. As we said previously, the types of vertexes we face in the

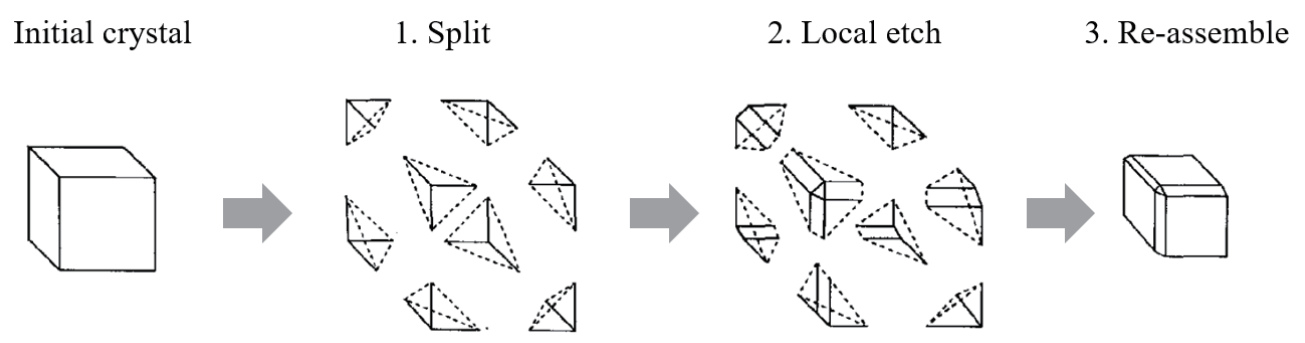

Fig. 1. An example of the split process. 
actual simulation are very complex: there are corner vertexes made up of three faces and corner vertexes made up of more faces; there are concave vertexes (the first line in Fig. 2), convex vertexes (the second line in Fig. 2), saddle vertexes (the third line in Fig. 2), and even false saddle vertexes (the fourth line in Fig. 2). It is relatively easy to distinguish the corner vertex with different numbers of faces. We consider primarily how to distinguish between concave, convex, saddle, and false saddle corner vertexes. The first two types can be composed of at least three faces, and the latter two types at least by four faces. The number of faces does not affect our ability to distinguish between them.

Figure 2 illustrates the resolution method. The etching direction in the figure is roughly from top to bottom (imagine the top of each corner is full of etching liquid). The slowness vectors in the figure refer to the speed etching vectors of a surface whose direction is the normal direction of the surface (in the opposite direction of the corrosive liquid) and whose magnitude is the reciprocal of the etching rate of this particular surface. Here, we simply focus on the fact that it characterizes the normal direction of a surface. In Fig. 2, we identified each of the faces at each corner in the order $a, b, c, d$. The second column gives the top view of the faces at each corner, and we can see that the order of faces is counterclockwise. The third column shows the slowness vectors for several faces at each corner, again from above. We found that the slowness vectors of concave and convex corners are still counterclockwise, whereas the vectors of the saddle corner are clockwise and the false saddle corner is mixed. From this, we can already distinguish three types of corners: saddle corner, false saddle corner, and concave and convex corners, the latter two being of the same type. Furthermore, we can simply distinguish between concave and convex. Concave and convex vertexes can be distinguished by whether the rotation direction of faces and the rotation direction of normal vectors are opposite.

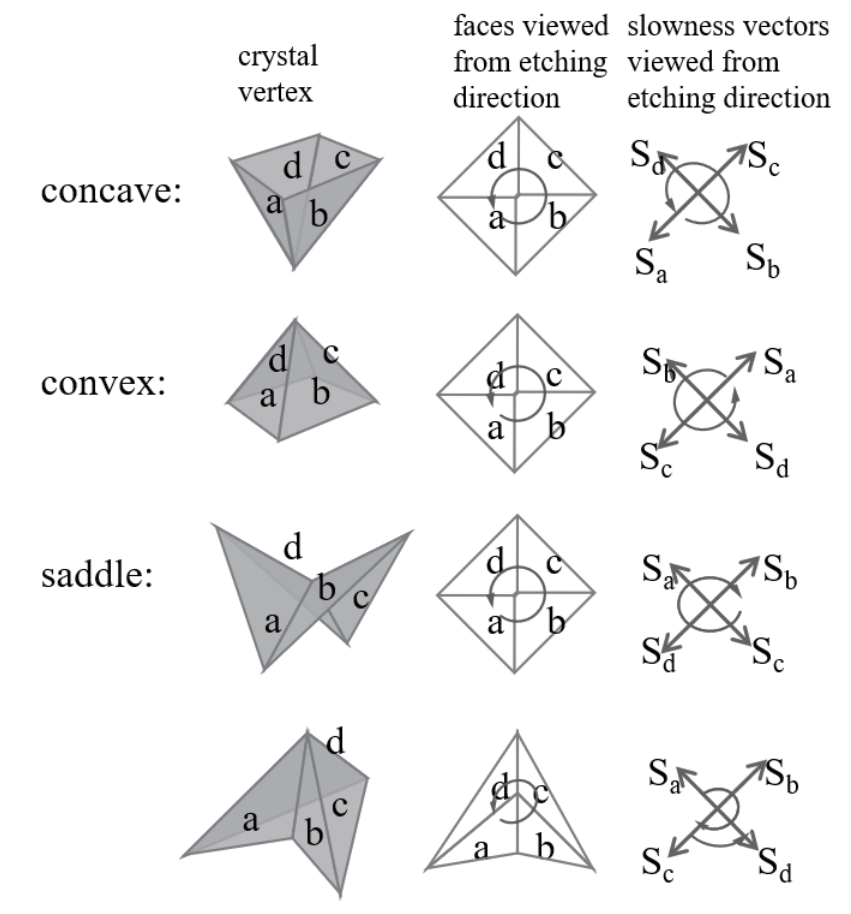

Fig. 2. Distinguishing corner types. 


\subsection{Method of processing a corner}

In this section, we show how to determine the evolution of etching in one time step in a 3D corner (Fig. 3). Here, we introduce the original 3D Hull method. For each vertex,

1. Draw slowness vectors $S_{a}, S_{b}$, and $S_{c}$ of the three faces $a, b$, and $c$ from the vertex point O.

2. Examine the slowness curve surface between the three slowness vectors $S_{a}, S_{b}$, and $S_{c}$.

3. Use a Hull construction to determine if there is any slowness vector on the surface of this curve that defines the emergence of a new face. In this particular case, no other slowness vectors define a new face, and the vertex point will not split.

4. Draw any new faces.

After we have calculated a 3D convexity in the graph using the 3D skinning method, we see that it has "shrunk", but it is still a sharp convex corner. In practice, the results of the calculation are most likely to be that a sharp corner turns into a flat hill made up of several convex corners.

\subsection{Method of processing an edge}

After vertex analysis, for each crystal vertex, analyze every edge and construct the proper inner or outer hull that defines the bevel face (Fig. 4); this is the same as the 2D Hull method.

\subsection{Method of determining the moving direction and distance}

The two previous sections do not specify how we determined the moving direction and the exact distance of each retracted surface consumed by the etching fluid. We describe that in detail here as shown in Fig. 5. Every single segment of the hull determines one moving

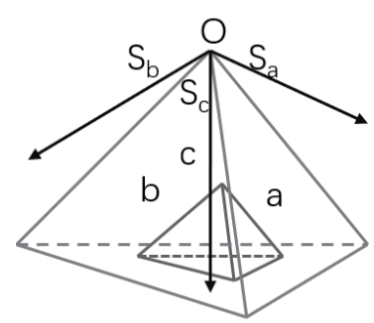

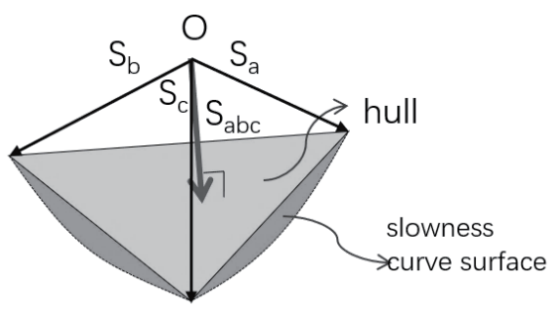

Fig. 3. Determining the evolution of etching in one time step in a $3 \mathrm{D}$ corner.

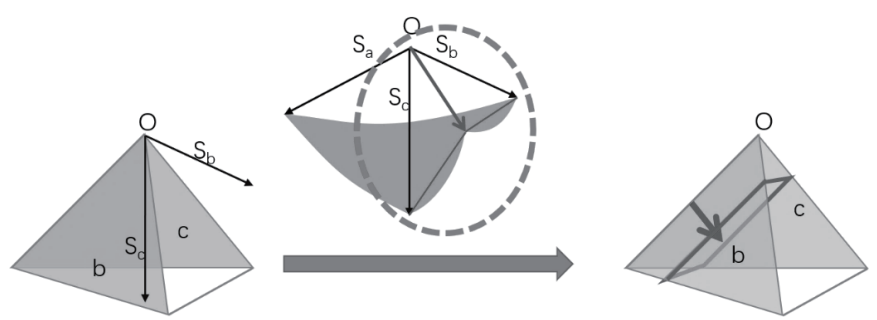

Fig. 4. Determining the evolution of etching in one time step on an edge. 


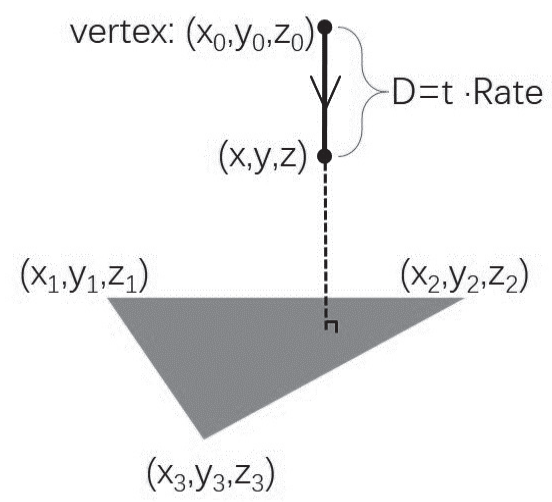

Fig. 5. Hull method determining the moving direction and the distance of a vertex.

direction of the vertex. The entire hull determines the splitting of the vertex. If a segment of the hull of a vertex is $\left(x_{1}, y_{1}, z_{1}\right),\left(x_{2}, y_{2}, z_{2}\right)$, and $\left(x_{3}, y_{3}, z_{3}\right)$, the verte $x\left(x_{0}, y_{0}, z_{0}\right)$ moves to $(x, y, z)$ in a time step ' $t$ ':

$$
x=x_{0}+\frac{D \cdot O}{\sqrt{O^{2}+P^{2}+Q^{2}}}, y=y_{0}+\frac{D \cdot P}{\sqrt{O^{2}+P^{2}+Q^{2}}}, z=z_{0}+\frac{D \cdot Q}{\sqrt{O^{2}+P^{2}+Q^{2}}},
$$

where

$$
\begin{aligned}
& D=t \cdot \text { Rate } \\
& O=\left(y_{2}-y_{1}\right)\left(z_{3}-z_{1}\right)-\left(z_{2}-z_{1}\right)\left(y_{3}-y_{1}\right), \\
& P=\left(z_{2}-z_{1}\right)\left(x_{3}-x_{1}\right)-\left(x_{2}-x_{1}\right)\left(z_{3}-z_{1}\right), \\
& Q=\left(x_{2}-x_{1}\right)\left(y_{3}-y_{1}\right)-\left(y_{2}-y_{1}\right)\left(x_{3}-x_{1}\right) .
\end{aligned}
$$

\section{Example of Simulation Results}

This simulator can simulate the etching process in a variety of situations. Here is an example. An experimental 3D Z-plate quartz crystal was etched with a square mask for $25 \mathrm{~min}$. The experimental conditions are as follows:

- Etchant: Ammonium bifluoride

- Concentration: Saturation

- Temperature: $82^{\circ} \mathrm{C}$

- Wafer: $Z$-plate (thickness $=100 \mu \mathrm{m}^{2}$ )

- Mask size: $200 \times 200 \mu \mathrm{m}^{2}$

- Etching time: $25 \mathrm{~min}$

- Time step: 1 min

The left of Fig. 6 shows experimental pictures of four corners taken with a scanning electron microscope. The right of Fig. 6 shows a simulation result with the same mask and the same etching time. In the top view, we measured the difference between the experimental and simulated values for the 25 corners (Table 1). The average of the differences is 3.8 degrees. The 

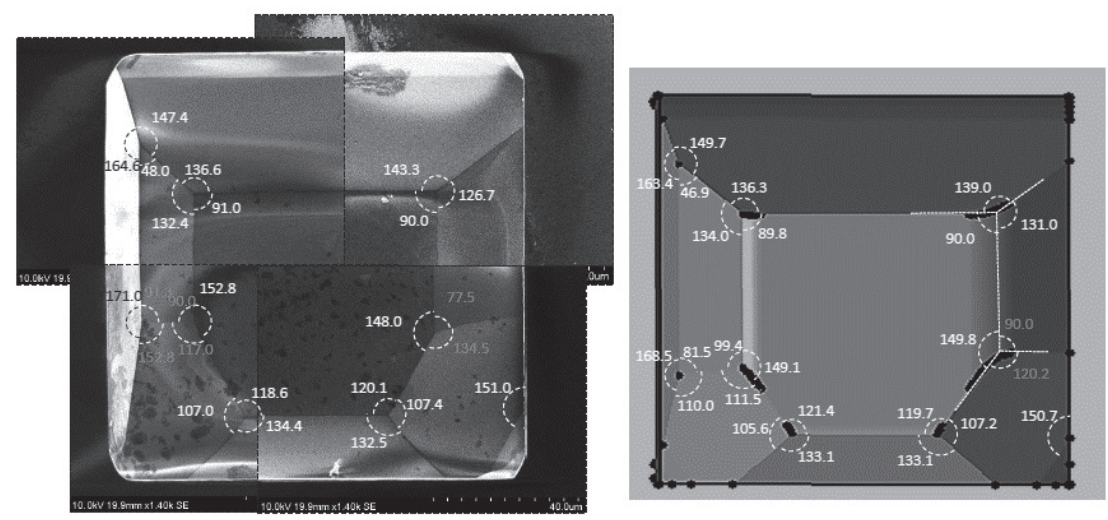

Fig. 6. 3D Z-plate square mask experimental result compared with simulation result.

Table 1

Summary of the experimental values.

\begin{tabular}{ccc}
\hline Experiment & Our simulator & Difference \\
\hline 147.4 & 149.7 & 2.3 \\
164.6 & 163.4 & -1.2 \\
48 & 46.9 & -1.1 \\
136.6 & 136.3 & -0.3 \\
132.4 & 134 & 1.6 \\
91 & 89.8 & -1.2 \\
171 & 168.5 & -2.5 \\
91.3 & 81.5 & -9.8 \\
97.7 & 110 & 12.3 \\
90 & 99.4 & 9.4 \\
152.8 & 149.1 & -3.7 \\
117 & 111.5 & -5.5 \\
107 & 105.6 & -1.4 \\
118.6 & 121.4 & 2.8 \\
134.4 & 133.1 & -1.3 \\
120.1 & 119.7 & -0.4 \\
107.4 & 107.2 & -0.2 \\
132.5 & 133.1 & 0.6 \\
151 & 150.7 & -0.3 \\
148 & 149.8 & 1.8 \\
77.5 & 90 & 12.5 \\
134.5 & 120.2 & -14.3 \\
143.3 & 139 & -4.3 \\
90 & 90 & 0 \\
126.7 & 131 & 4.3 \\
\hline & &
\end{tabular}

two places where we find the greatest contribution to the simulation error are curves, if the two curves are not taken into account. The average of differences will be smaller. We can see that the simulation result, including the etching shape and the angle values, relatively reproduces the experimental result perfectly. 


\section{Conclusion}

In this article, we describe in detail our theoretical achievements in the work of simulating anisotropic crystal etching, especially the breakthrough progress in key points such as the resolution method, corner type resolution, corner processing, and edge processing. On the basis of these theories, we have developed simulation systems that work well, and in future articles, we will describe more the simulation system and results. This work is an original theoretical work. In the process of transforming the physical language of the etching process into mathematical language, the authors consider that there is still much room for improvement on the more accurate determination of etching rate database, easy-to-use 3D simulation interface development and optimization, and the effect of simulation time steps on the accuracy of the results. Our team will also explore these issues in future work.

\section{Acknowledgments}

This work was supported by the National Natural Science Foundation of China (Grant No. 1702251) and the Fundamental Research Funds for the Central Universities (Grant Nos. JBX170415 and JBX170410).

\section{References}

1 F. C. Frank and M. B. Ives: J. Appl. Phys. 31 (1960) 1996.

R. J. Jaccodine: J. Appl. Phys. 33 (1962) 2643.

C. H. Sequin: Sens. Actuators, A 34 (1992) 225.

4 C. Tellier, N. Vialle, and J. Vaterkowski: 40th Annu. Symp. Frequency Control (1986) 76.

5 C. R. Tellier, N. Vialle, and J. L. Vaterkowski: Surf. Coat. Technol. 34 (1988) 417.

6 K. Sato, K. Asaumi, G. Kobayashi, Y. Iriye, and M. Shikida: IEICE Trans. J82-C-II 3 (1999) 84 (in Japanese).

7 D. Cheng, K. Sato, M. Shikida, A. Ono, K. Suto, K. Asaumi, and Y. Iriye: Proc. 2003 Int. Symp. Micromechatronics and Human Science (2003) 281.

8 R. A. Buser and N. F. de Rooij: Sens. Actuators, A 28 (1991) 71.

9 J. Fruhauf, K. Trautmann, J. Wittig, and D. Zielke: J. Micromech. Microeng. 3 (1993) 113.

10 U. Heim: J. Micromech. Microeng. 3 (1993) 116.

11 S. Osher and J. A. Sethian: J. Comput. Phys. 79 (1988) 12.

12 C. H. Sequin: Sens. Actuators, A 34 (1992) 225.

13 M. Zhao, J. N. Wang, H. Oigawa, J. Ji, H. Hayashi, and T. Ueda: IEEJ Trans. Sens. Micromach. 131 (2011) 185.

\section{About the Authors}

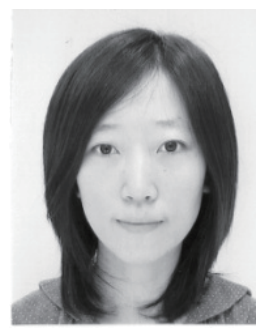

Meng Zhao received her B.Sc. degree in Physics from Nankai University, China, in 2003. She received her Ph.D. degree in astrophysics from the Graduate University of the Chinese Academy of Sciences in 2009. From 2010 to 2015, she worked as a research associate and researcher at the Information, Production, and Systems Research Center, Waseda University, Japan. Since 2016, she has been an associate professor at Xidian University, P.R. China. She engages in research and development of anisotropic wet etching simulators for quartz crystals. 


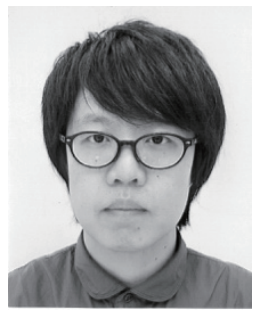

Jing Ji received his B.Sc. degree in Microelectronic Engineering from Nankai University, China, in 2003, and his M.S. and Ph.D. degrees in engineering of information, production, and systems from the Graduate School of Information, Production, and Systems, Waseda University, Japan, in 2007 and 2013, respectively. From 2013 to 2016, he worked as a research associate and researcher in the Information, Production, and Systems Research Center, Waseda University, Japan. Since 2016, he has been an associate professor at Xidian University, P.R. China. He is a member of the Institute of Electrical and Electronics Engineers and the Institute of Electrical Engineers of Japan. His research area includes quartz MEMS devices, finite element methods, and sensing technology.

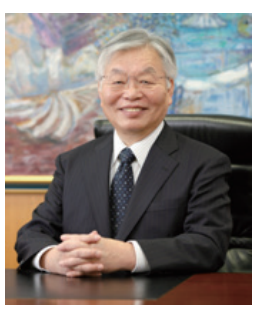

Toshitsugu Ueda received his B.E. and M.E. degrees in electrical engineering from Shinshu University, Nagano, Japan, in 1969 and 1971, respectively. He received his Ph.D. degree from Tokyo Institute of Technology in 1988. Since joining Yokogawa Electric Corporation in 1971, he has been engaged in developing low-noise amplifiers, mechanical resonators, micromachining technologies, and sensors using these technologies to control temperature, pressure, and displacement. He has been a professor of the Graduate School of Information, Production, and Systems (IPS), Waseda University since 2003 and Dean of IPS and IPS center in 2012. He has received awards from the Society of Instrument and Control Engineers (Japan) in 1987 and 1994, as well as from the Japan Institute of Invention and Innovation in 1985 and 1987. He is a fellow of the Society of Instrument and Control Engineers (Japan). He is a member of the Institute of Electrical Engineers (Japan) and the Society of Instrument and Control Engineers (Japan). 